TTR

Traduction, terminologie, rédaction

\title{
La boîte noire de l'historiographie interculturelle belge. Les allers-retours d'Emma Lambotte entre Liège et Anvers (1895-1950)
}

\section{Maud Gonne}

Volume 31, numéro 2, 2e semestre 2018

Minorité, migration et rencontres interculturelles : du binarisme à la complexité

Minority and Migrant Intercultural Encounters: From Binarisms to Complexity

URI : https://id.erudit.org/iderudit/1065573ar

DOI : https://doi.org/10.7202/1065573ar

Aller au sommaire du numéro

Éditeur(s)

Association canadienne de traductologie

ISSN

0835-8443 (imprimé)

1708-2188 (numérique)

Découvrir la revue

Citer cet article

Gonne, M. (2018). La boîte noire de l'historiographie interculturelle belge. Les allers-retours d'Emma Lambotte entre Liège et Anvers (1895-1950). TTR, 31(2), 167-194. https://doi.org/10.7202/1065573ar
Résumé de l'article

L'historiographie traditionnelle a eu tendance à réduire la complexité de la nation belge à l'interaction (conflictuelle) entre communautés francophone et néerlandophone (ou flamande). Cette approche binaire a marginalisé la pluralité d'un territoire national diglossique fragmenté en parlers flamands, wallons et germaniques, où seule une élite minoritaire utilisait le français. Dans cette configuration asymétrique, les échanges, déplacements et traductions entre les différentes cultures minoritaires révèlent des dynamiques bien plus complexes que la seule confrontation entre une langue dominante et une langue dominée. En se penchant sur les allers-retours d'Emma Lambotte (1876-1963) - peintre, auteure, traductrice, chroniqueuse, salonnière et activiste wallonne - entre Anvers et Liège, cet article tâche d'entrouvrir la boîte noire de l'historiographie interculturelle belge via l'introduction inédite d'une perspective minoritaire wallonne. 


\title{
La boîte noire de l'historiographie interculturelle belge. Les allers-retours d'Emma Lambotte entre Liège et Anvers (1895-1950)
}

\author{
Maud Gonne \\ F.R.S. - FNRS \\ Université de Namur/Université catholique de Louvain
}

\begin{abstract}
Résumé
L'historiographie traditionnelle a eu tendance à réduire la complexité de la nation belge à l'interaction (conflictuelle) entre communautés francophone et néerlandophone (ou flamande). Cette approche binaire a marginalisé la pluralité d'un territoire national diglossique fragmenté en parlers flamands, wallons et germaniques, où seule une élite minoritaire utilisait le français. Dans cette configuration asymétrique, les échanges, déplacements et traductions entre les différentes cultures minoritaires révèlent des dynamiques bien plus complexes que la seule confrontation entre une langue dominante et une langue dominée. En se penchant sur les allers-retours d'Emma Lambotte (1876-1963) - peintre, auteure, traductrice, chroniqueuse, salonnière et activiste wallonne - entre Anvers et Liège, cet article tâche d'entrouvrir la boîte noire de l'historiographie interculturelle belge via l'introduction inédite d'une perspective minoritaire wallonne.
\end{abstract}

Mots-clés : Wallonie, minorité, Emma Lambotte, traduction, boîte noire, théorie de la complexité

Abstract
Traditional historiography has tended to reduce the complexity of the
Belgian nation to the (conflictual) interaction between French and Dutch
(or Flemish) communities. This binary view, however, systematically mar-
ginalized the plurality of a diglossic national territory fragmented into
Flemish, Walloon, and German variants, where only a minority elite spoke
French. The exchanges, travels and translations between the different mi-
nority cultures within this asymmetrical configuration reveal a much more
complex dynamics than the mere confrontation between a dominant and
a dominated language. Focusing on Emma Lambotte's (1876-1963) trips 
between Antwerp and Liège-as a Walloon painter, writer, translator, chronicler, salonnière and activist-this article aims to open the black box of Belgian intercultural historiography by introducing a unique Walloon minority perspective.

Keywords: Wallonia, minority, Emma Lambotte, translation, black box, complexity theory

\section{Introduction}

Considérée comme l'archétype du carrefour culturel, où les mouvements, médiations et compromis entre langues germaniques et romanes sont plus visibles que nulle part ailleurs (Leerssen, 2014, p. 1395), la Belgique est un exemple classique pour l'étude des relations interculturelles, des échanges littéraires et des traductions. La plupart de ces recherches se sont intéressées à l'interaction plus ou moins conflictuelle entre francophones et néerlandophones, qui n'a cessé de monopoliser la scène politique belge jusqu'à nos jours ${ }^{1}$. Selon la version historique en vigueur, l'espace linguistique et culturel belge aurait en effet été caractérisé, depuis l'indépendance en 1830, par une diglossie franco-flamande : un peuple flamand pratiquant les dialectes se serait progressivement opposé à une élite francophone au pouvoir au cours d'un long processus d'émancipation qui aurait finalement mené au tracé d'une frontière linguistique en 1962, et au principe de monolinguisme territorial.

Il y aurait pourtant lieu de dépasser cette vision binaire qui semble justifier, plutôt que d'expliquer, l'actuelle configuration de la Belgique ${ }^{2}$. Jusqu'au-delà de la Deuxième Guerre mondiale, le contexte culturel et linguistique belge est bien plus complexe que la seule confrontation entre une langue dominante (le français) et une langue dominée (le flamand). Derrière une illusion de binarité, l'historiographie nationale a marginalisé la pluralité d'un territoire hétérogène, fragmenté en dialectes flamands, wallons et allemands,

1 Voir entre autres De Geest et Meylaerts (2004) et Buelens (2011).

2. C'est-à-dire un pays "divisé», avec le néerlandais comme langue officielle au nord, le français comme langue officielle au sud et un bilinguisme officiel francoflamand dans la région de Bruxelles-capitale. Il faut également compter sur une minorité germanophone à l'est. Pour plus d'informations sur la configuration politico-linguistique de la Belgique, y compris sur les compétences attribuées aux régions (flamande, wallonne, bruxelloise) et communautés (flamande, germanophone et francophone, rebaptisée fédération Wallonie-Bruxelles en 2011), consulter le site officiel Belgium.be (s. d.). 
où seule une élite minoritaire parlait le français ${ }^{3}$. Comme l'affirme le sociolinguiste Michel Francard :

Au moment de l'indépendance de la Belgique, la majorité de la population wallonne ne parle pas français, mais une langue régionale romane (wallon, picard, lorrain, champenois) ou germanique. De ce point de vue, on l'ignore parfois, Wallons et Flamands des classes populaires et des régions rurales se sont vu imposer, par la bourgeoisie francophone qui a cimenté l'État belge, une langue différente des parlers d'une majorité de la population. (1998, p. 16)

Cette politique linguistique s'est avérée, au départ, peu problématique pour une population qui s'identifiait alors à une communauté locale - où les dialectes étaient les langues véhiculaires - et à une classe sociale, plutôt qu'à une communauté linguistique (Meylaerts, 2004 , p. 35). Il n'empêche qu'un siècle et demi plus tard, trois langues - et force est de constater, exogènes ${ }^{4}$ - ont acquis un statut officiel sur base territoriale : le français, le néerlandais et l'allemand. Dans cette transition d'un monolinguisme national de fait vers un trilinguisme régional officiel, la relation de la Wallonie avec les cultures, langues et agendas identitaires voisins reste terra incognita.

Alors que la marginalisation de la composante germanophone, qui a longtemps souffert d'une association avec la Prusse puis avec l'Allemagne belligérantes, dans le panorama national semble peu à peu se résorber ${ }^{5}$, la faille historiographique wallonne demeure béante. À tel point que le peu d'études qui se sont attelées à la question d'une identité culturelle wallonne ont qualifié cette dernière de "faible», "aliénée », "peu sûre d'elle-même, sans doute» (Courtois et Pirotte, 1994 , p. 276), en comparaison à une identité flamande «forte» (Lecours, 2006, p. 51). Victime d'un complexe d'infériorisation et de

3. Dès les premières décennies de l'indépendance, et bien que la liberté linguistique fût inscrite dans la Constitution, la langue française, vecteur de progrès et d'ouverture internationale, s'impose comme seule langue officielle tandis que l'État belge entame un processus d'éradication des dialectes.

4. Ceci mène Michel Francard (2013, p. 55) à parler d'un pays de «coupeurs de langues»: Wallons et Flamands ont choisi une langue exogène comme langue officielle. Plutôt que de promouvoir le flamand ou le wallon - ce qui aurait nécessité la création d'une langue commune au départ des diversités dialectales flamandes et wallonnes -, les choix se sont portés sur des langues standardisées, bien que ressenties comme étrangères : le français de France en Wallonie, et le néerlandais des Pays-Bas en Flandre. En adoptant l'allemand standard, la communauté germanophone en a fait de même.

5. Voir notamment Beck et Roland (2013). 
minorisation, «la Wallonie ne déclenche pas chez celui qui l'entend l'évocation d'une imagerie homogène, ne provoque pas la référence à une évidence massive, indiscutée» (Klinkenberg, 1988, n. p.).

C'est sur l'incorporation de la minorité wallonne dans l'histoire interculturelle constitutive de l'espace belge que se penchera cet article. Cette intégration inédite modifie considérablement le tableau : elle nous invite d'une part à reconsidérer les idées développées autour d'une configuration «binaire» (français versus flamand) et, d'autre part, à nous interroger sur les modalités de réussite et d'échec du développement des minorités culturelles. La Wallonie a (presque ${ }^{6}$ ) systématiquement été étudiée en vase clos, comme si, contrairement à ses voisines, elle s'était auto-organisée, sans rapport avec son environnement. Or, la minorité est une relation, et non une essence (Cronin, 1995, p. 86). En partant du principe que la reconnaissance de cultures émergentes dépend en grande partie d'échanges interculturels dans les domaines de l'art et de la littérature (Lobbes et Gonne, 2014), la question wallonne sera ici abordée à partir de la perspective des réseaux, de la médiation et de la traduction, laquelle, «contrairement à une image largement répandue, [...] ne tient pas toujours un rôle bénéfique émancipatoire pour les minorités» (Meylaerts, 2009, p. 12).

Il ne s'agira pas d'écrire une histoire au conditionnel ou d'hypostasier, mais de dépasser l'étude des vainqueurs de l'histoire culturelle belge pour examiner le processus de développement culturel de la Wallonie. En suivant le parcours inédit, entre Liège et Anvers, d'Emma Lambotte (1876-1963), une intellectuelle belge qui a travaillé dans la première moitié du $\mathrm{XX}^{\mathrm{e}}$ siècle à la promotion d'une culture wallonne, cette étude tâchera de révéler les modalités d'un échec (inter)culturel.

\section{Bref aperçu d'une quête identitaire manquée}

Dans la deuxième moitié du XIX ${ }^{\mathrm{e}}$ siècle, on enregistre en Wallonie une quête identitaire typique, comparable à celle qui a lieu en Flandre à la même époque. Cette quête est menée par une série d'hommes et de femmes de lettres, qui réfléchissent à l'articulation du wallon, du flamand et du français en Belgique et tentent de construire et de diffuser un répertoire culturel wallon à l'intérieur, d'abord, puis

6. Voir par exemple Balace (2012). 
à l'extérieur des frontières régionales ${ }^{7}$. Ces agents de transfert ${ }^{8}$, ou médiateurs, favorisent la prolifération d'études sur l'originalité wallonne et la publication d'anthologies de littérature wallonne (p. ex. Sottiaux, 1906; Haust, 1924). Ils commanditent des expositions sur l'art wallon, créent des symboles (hymne wallon, drapeau wallon, fêtes de Wallonie), fondent des organes de presse (p. ex. L'Âme wallonne, Wallonia), des sociétés et des rassemblements culturels et littéraires (p. ex. les Congrès wallons, la Société de langue et littérature wallonnes). Mais ce mouvement se tourne également vers les cultures avoisinantes en promouvant notamment la traduction de la littérature wallonne vers l'allemand, le néerlandais et le français, et vice versa $a^{9}$.

En constante interaction avec le français, langue des lettrés, et les variantes flamandes et germaniques, les parlers wallons connaissent alors une vitalité qui dépasse largement la sphère littéraire. C'est bien simple, jusqu'en 1920, $80 \%$ des Wallons préferent utiliser le wallon dans leurs contacts avec l'administration ${ }^{10}$. Aussi, la première loi linguistique significative votée en Belgique, la loi d'égalité de 1898, qui met officiellement les langues française et flamande sur un même pied, est ressentie comme un énorme échec ${ }^{11}$. Dans la revue L'Áme wallonne du 8 mars 1898, on peut lire :

Est-ce nosse langue çoula? Nenni, c'est l'Wallon. (Rimarquez qu ji n'dispréhe nin n'gotte li Français, qui n's estans firs dè k'nohe et qu'est jâsé d'tos costés)

7. «In each group, there is a small minority who acts as producers on the level of the repertoire itself. Whether entrusted by the group with the task of doing so or whether self-appointed, it is mainly those persons who introduce new options, and hence act as agent of change» (Toury, 2002, p. 151).

8. Les transferts culturels désignent le passage et la transformation d'objets, de textes et d'idées d'un contexte culturel ou linguistique à un autre (Espagne, 2013). Ces transferts sont sensibles à la réciprocité, au croisement et à l'imbrication; ils impactent toutes les entités (personnes, pratiques ou objets croisés) impliquées et transformées dans le processus (Werner et Zimmerman, 2003).

9. Notons entre autres les traductions de Molière par Henri Simon (1912) et Bosquetia (1907); les traductions de Remouchamps (1910,1934) et Simon (1914) vers le français par Jean Haust; les traductions de Defrêcheux vers le néerlandais par August Cuppens (1900), et vers l'allemand par Albert Wachter (1908).

10. Selon une enquête de J-M. Remouchamps menée en 1920. Les résultats de cette enquête sont repris dans Francard (2013, p. 51).

11. Pour plus d'information sur la loi de 1898, lire D'hulst et al. (2016). 
[Est-ce notre langue celle-là (le français)? Non, c'est le wallon (remarquez que je ne méprise pas le français, que nous sommes fiers de connaître, et qui est parlé partout) (ma trad.)].

D'autant que, l'année précédente, la glorification de l'Âme belge par Edmond Picard - syncrétisme romantique de la langue française et de la race flamande (Roland, 2004) - avait également exclu la composante wallonne du projet national. Suite au vote de cette loi qui stipule l'équivalence du français et du néerlandais dans la législation belge, la Wallonie, qui tenait alors les rênes de l'économie belge grâce à son industrie florissante, demande pour la première fois une séparation administrative de la Belgique, sans résultat. La suite de l'histoire est un ensemble d'échecs et de vœux sans lendemain ${ }^{12}$ concernant notamment l'enseignement du wallon et de l'histoire de la Wallonie, la création d'une académie wallonne commanditée par l'État, l'utilisation de traducteurs wallons dans les tribunaux, un bilinguisme franco-wallon dans l'administration, l'annulation de la loi de 1898 et une nouvelle demande de séparation en 1912. Ils sont de plus en plus nombreux à voir dans les victoires successives du mouvement flamand une menace d'assimilation culturelle et identitaire :

Flamingants honteux, des Flamands égoïstes, des mangeurs de Wallonie, qui croient, comme le Parisien, - o dérision! - que Paris c'est la France et la Flandre à elle seule forme toute la Belgique [...] frères wallons, prenez-garde! On vous vole! (Debouck, 1909, p. 214)

Après la guerre, les Wallons se tournent progressivement vers la France et le français. Le programme culturel et linguistique est peu à peu abandonné pour laisser place à un mouvement politique antiflamingant ${ }^{13}$. L'enseignement devenu obligatoire, les écoles publiques imposent une éducation en langue française à tous les enfants de Wallonie, ce qui engendre un dénigrement des dialectes. Le climat socioéconomique et international qui suit la Seconde Guerre mondiale sonne le glas de la transmission intergénérationnelle du wallon et entame le déclin économique de la Wallonie.

Cet aperçu trop bref engendre de multiples questions. Du point de vue (inter-)culturel, les interventions de médiateurs au niveau

12. Pour plus d'information sur les vœux avortés du mouvement wallon, lire le compte-rendu du Congrès wallon de 1905.

13. Le terme "flamingant» désigne un partisan du mouvement flamand, une personne qui défend la langue et la culture flamandes et s'oppose à l'influence du français en Belgique. Le terme revêt une connotation péjorative. 
local (traductions, anthologies, créations d'institutions) n'ont pas résulté en une identité wallonne forte, mais en un agrégat complexe en manque de légitimation. Comment deux quêtes similaires - la quête wallonne et la quête flamande - qui se sont déroulées presque simultanément dans un même espace national, ont-elles pu résulter en des configurations si différentes? Pour appréhender ces questions, il nous faut interroger la «boîte noire» et entrer dans le paradigme de la complexité (Morin, 1990).

\section{Input, output et black-box}

Pour le philosophe Edgar Morin, le paradigme de la simplicité consiste à considérer les entrées dans le système (inputs) et les sorties (outputs), sans entrer toutefois dans le mystère de la boîte noire (2005, p. 49).

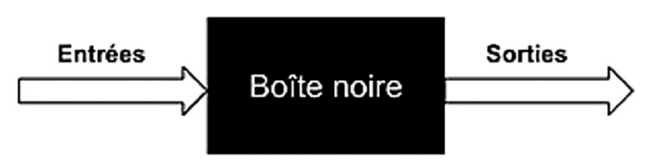

Figure 1. Schématisation d'une boîte noire (Moyaux, 2018, n. p.)

Dans le cas de la transition linguistique et culturelle en Belgique, l'évolution d'une configuration d'entrée complexe (situation linguistique et culturelle hétérogène) vers une configuration de sortie simple (divisions linguistiques territoriales) n'est que partiellement connue. Elle a été étudiée à la lueur de la sortie, marginalisant par conséquent la composante wallonne et, avec elle, le long processus de réduction et de sélection, donnant ainsi «à croire que le découpage arbitraire opéré sur le réel était le réel lui-même» (ibid., p. 19).

La complexité, en revanche, tente d'explorer les nouveaux défis d'un monde connecté dans lequel les effets ne sont plus directement relié à des causes et dans lequel la dynamique d'input-output (et de feedback, ou boucle de rétroaction) est imprévisible. Elle aspire à identifier les systèmes et processus qui «lack the order and stability required to produce universal rules about behaviour and outcomes » (Cairney, 2012, p. 347) en laissant la place aux incertitudes, paradoxes et échecs. La théorie de la complexité veut ainsi compléter les approches «de la simplicité » qui tendent à chasser le désordre en isolant, réduisant et opposant les entités plutôt qu'en les reliant (complexus $=$ tisser ensemble). La complexité ne s'intéresse pas au 
résultat final et légitime, mais au processus de connexion, à l'émergence, ou, autrement dit, à la façon dont les relations entre parties donnent naissance à des systèmes complexes, qui tentent de s'adapter à leur environnement en atteignant un équilibre productif :

component parts interact to form complex systems that display new characteristics as a result of their complex interactions. The new and possibly unique emergent properties define new entities. (Chamberlin, 2009, p. 93)

Selon cette logique, des composantes similaires ou comparables par exemple, l'input des mouvements minoritaires flamand et wallon pour la promotion de leur langue et culture dans le cadre national belge - n'interagissent pas de la même façon et ne risquent donc pas de générer le même résultat. Le système émergent, s'il ne s'adapte pas à son environnement, peut tout simplement disparaître ou ne pas atteindre le résultat escompté par les parties. Comme le résume Reine Meylaerts :

Nonlinear change or nonlinear causality means that similar causes need not lead to similar results: small differences in initial conditions may exert major influences on the eventual results. Much comes from little or sometimes nothing comes from very much. The determination of causation is bidirectional or complex: from the local to the global to the local, that is, upward and downward causation. (2017, p. 50)

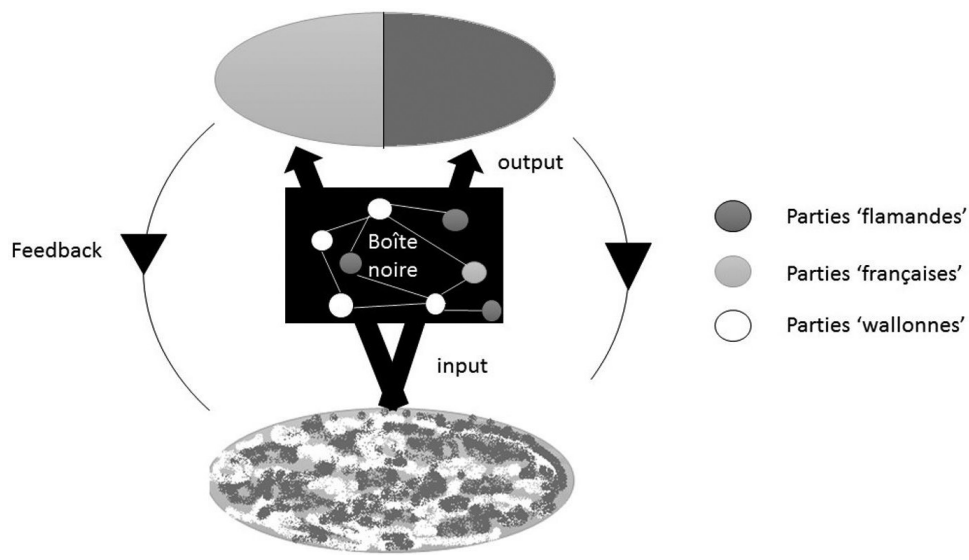

Figure 2. Les relations interculturelles belges en tant que système complexe 
Dans cette optique, le concept de boîte noire - issu de la cybernétique (Wiener, 1952) - échappe à la causalité linéaire : on connaît le comportement de la boîte, mais pas son fonctionnement, qui est inaccessible, invisible ou délibérément omis. C'est un concept-outil qui met en évidence l'entropie d'un système et son autorégulation (par le biais de la boucle de rétroaction qui relie causes et effets ${ }^{14}$ ). Dans le cadre d'une quête identitaire wallonne manquée et effacée derrière une illusion de binarité, la boîte noire peut désigner un réseau de relations (sociales, discursives, politiques, artistiques, etc.) ayant favorisé l'émergence - mais pas la pérennité - d'une identité culturelle. Or, une façon d'aborder ces relations consiste à suivre les agents «invisibles» de la quête : les médiateurs et traducteurs qui, par définition, relient langues, cultures, identités, et ce, suivant des objectifs individuels et collectifs. Ils agissent localement, créent des réseaux, orchestrent des transferts et véhiculent des représentations. Femme de lettres, salonnière, peintre, conférencière, traductrice, essayiste, biographe et militante wallonne, Emma Lambotte s'est trouvée au centre de nombreuses activités interculturelles locales. En incarnant un enchevêtrement de rôles médiateurs et d'agendas identitaires complexes, voire paradoxaux, elle nous offre un accès privilégié à la boîte noire.

\section{3. Étude de cas. Les allers-retours d'Emma Lambotte entre Liège et Anvers}

Les villes sont des espaces privilégiés de négociation et de résistance culturelles et linguistiques; des sites où les discours identitaires sont débattus, diffusés et traduits. Elles portent les traces de la cohabitation de langues et d'institutions asymétriques (Cronin et Simon, 2014). Dans la deuxième moitié du XIX ${ }^{\mathrm{e}}$ siècle, Liège et Anvers sont deux grandes métropoles internationales et multilingues.

Jusqu'à 1789, Liège était la capitale de la principauté de Liège, un état du Saint Empire romain recouvrant des parties des actuelles Wallonie et Flandre. Ce n'est que sous l'Empire français, puis hollandais que se confondent les destins de l'ancienne principauté et du reste de l'actuelle Belgique. À partir des années 1850, Liège abrite une des plus grandes industries métallurgiques au monde et se place aux premières lignes de la révolution industrielle. Son économie florissante attire des travailleurs, ingénieurs, professeurs et étudiants de toute la Belgique ainsi que d'Europe (Raxhon, 2004). La ville

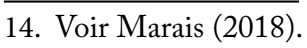


devient également le terreau d'un mouvement culturel qui aspire à la redécouverte (ou réinvention) et à la promotion d'un héritage culturel et littéraire wallon. La diglossie et le multilinguisme font partie intégrante de paysage urbain (Gross, 2001) : le français, lingua franca, est pratiqué aux côtés d'une variété de dialectes wallons, flamands et germaniques.

L'histoire d'Anvers, berceau du mouvement flamand (Luyckx et Platel, 1985), est pour sa part liée à celle des Pays-Bas bourguignons, espagnols puis autrichiens. Ville portuaire et principale métropole de Flandre, Anvers baigne depuis le XVII e siècle dans la coprésence de nationalités, cultures et langues. Les classes nobles et bourgeoises de la ville adoptent graduellement la langue véhiculaire européenne, le français, tandis que la majorité de la population locale parle les dialectes flamands et wallons. Au début de la deuxième moitié $\mathrm{du} \mathrm{XIX}^{\mathrm{e}}$ siècle, la classe moyenne anversoise - largement bilingue franco-flamand - s'attelle, avec succès, à l'émancipation du flamand à Anvers, en Flandre, en Belgique et même à l'étranger (Meylaerts et Gonne, 2014).

\section{De Liège (1876-1892)...}

Emma Lambotte, née Protin, voit le jour à Liège le 11 août 1876 dans une famille aisée d'imprimeurs. Elle appartient à l'élite liégeoise bilingue (franco-wallonne). Son père, Édouard Protin, meurt en 1884, laissant trois orphelins : Juliette, Robert - qui deviendra champion du monde de cyclisme sur piste et reprendra l'imprimerie familiale - et Emma, la cadette. Jeune fille, elle commence ses études à l'Institut du Nord puis s'inscrit au lycée liégeois de la Baronne Léonie de Waha de Chestret (1836-1926), une pédagogue féministe et fervente militante wallonne, qui offre un programme de qualité aux jeunes filles ${ }^{15}$. Grande intellectuelle et fondatrice de l'Union des femmes de Wallonie, dont Lambotte assumera plus tard la présidence, Waha défend l'enseignement de l'histoire ${ }^{16}$, de la langue et de la culture wallonnes, et propose des solutions séparatistes aux questions linguistiques belges dans une rhétorique imprégnée

15. Léonie de Waha a créé le premier établissement scolaire (non catholique) formant les jeunes filles à l'Université.

16. L'enseignement officiel a toujours marginalisé l'histoire de la Wallonie, et en particulier celle de la principauté de Liège. La sélection de l'histoire des provinces flamandes, à partir de l'unification des Pays-Bas bourguignons au XVe siècle, assurait en effet une plus grande homogénéité nationale et justifiait le bilinguisme et biculturalisme belges (voir Hasquin, 1982). 
de romantisme racial : «Oui, le caractère de la race persiste; nous ne sommes pas et nous ne serons jamais des bastardés Flaminds [Flamands abâtardis; ma trad.]» (Waha, citée dans Lambotte, 1927, p. 128). Emma glorifiera cette "grande Wallonne des temps révolus " (ibid., p. i), qu'elle juge pleine d'humour et de sagesse, dans un essai biographique en 1927.

Des ennuis de santé forcent la jeune fille à abandonner son parcours scolaire; elle suit alors quelques cours particuliers à l'Académie des Beaux-Arts de Liège et finit ses études dans un pensionnat religieux. À 18 ans, elle épouse le docteur Albin Lambotte, de dix ans son aîné, un chirurgien de renommée internationale, passionné d'art et fixé à Anvers. La jeune Wallonne abandonne les rives de la Meuse pour celles de l'Escaut et déménage dans la capitale du mouvement flamand.

\section{... à Anvers (1895-1918)}

Dans la métropole anversoise, Emma sort, assiste aux concerts avec Albin, prend le thé, organise des salons, donne des dîners; mais sous le pseudonyme d'Emaël, elle peint, écrit et s'adonne à la critique littéraire et artistique. Elle s'érige en femme indépendante et cultivée, qui plus est grande escrimeuse ${ }^{17}$. Tandis que dans la ville grondent les tensions entre Flamands, francophones et Wallons, cette salonnière hors pair réunit, dans son hôtel particulier rue Louise, des écrivains et artistes de tous horizons ${ }^{18}$. On y voit des écrivains étrangers comme Guillaume Apollinaire, Jehan Rictus, Laurent Tailhade et Neel Doff ainsi que des écrivains flamands et wallons comme Max Elskamp, Émile Verhaeren, Albert Mockel et Maurice Maeterlinck. On y croise également des militants wallons philologues tel Jules Feller ou politiciens tel Louis Pierard, ainsi que des artistes peintres comme le flamand James Ensor et les Wallons Auguste Donnay et Armand Rassenfosse - qui illustreront plusieurs de ses recueils. C'est évidemment le français qui est de mise dans cet environnement artistique et intellectuel raffiné. Dans un vibrant hommage, Maurice Gauchez rappelle l'ambiance qui régnait alors dans le microcosme crée par les Lambotte :

Emile Verhaeren, au cours de quelques jours passés à Paris, en 1914, nous avait rappelé que lui-même avait été reçu chez le docteur

17. Elle assure la présidence du Ladies Fencing Club de 1907 à 1914.

18. Voir Derrey-Capon (1999) et les correspondances d'Emma Lambotte conservées aux AMVC de la Letterenhuis d'Anvers, sous la cote L 1604. 
Lambotte, comme Albert Mockel, comme le philologue Feller, et, lorsque nous nous attardions, boulevard de Belgique, auprès de Max Elskamp, souvent l'un ou l'autre nous parlait d'elle, créature séduisante, spirituelle, cultivée et... présidente de l'Union des femmes de Wallonie. ${ }^{19}$

Parmi les contacts de son réseau, il faut mentionner François Franck, collectionneur et mécène anversois, actif dans les réseaux artistiques du mouvement flamand. Malgré certains différends idéologiques, Emma et François se vouaient une admiration mutuelle : "Nous étions d'accord sur tous les points, un seul excepté : la question flamande. Aussi évitions-nous d'en parler craignant de nous peiner l'un l'autre» (Lambotte, 1933, citée dans Derrey-Capon, 1999, p. 39). Elle fut la première à lui présenter le peintre James Ensor. C'est grâce à son entremise qu'Ensor se forgera une position de choix sur la scène belge :

Franck, Anversois blond et puissant, Emaël, Liégeoise, brune et ténue, vous vous êtes ligués pour me défendre. Ô! La belle alliance, le touchant symbole, le gage d'entente, de paix et de fraternité. Anvers et Liège, les deux pôles rivaux que j'aime. (Ensor, 1933, cité dans Derrey-Capon, 1999, p. 37)

En contrepartie, Ensor permet à Lambotte d'exposer quelques-unes de ses œuvres au Kursaal d'Ostende en 1908.

Linaccessible Emma séduit. Ils sont nombreux à chercher la compagnie de cette «Ève sûre d'elle-même face à l'Adam soumis » (Gauchez, 1952 $2^{20}$ ), "Liégeoise d'élite au cœur vibrant, amie fidèle et sensible, magicienne éprise d'art libre! Libre!! Libre!!! Encore plus libre»(Ensor, cité dans Derrey-Capon, 1999, p. 24). Dans leurs échanges épistolaires, les écrivains Albert Mockel se perdent en compliments, excuses et marques d'affection, tandis que Max Elskamp se fait appeler «son mandarin ${ }^{21}$. Deux grands peintres belges, Henri de Groux et James Ensor, se disputent même son portrait en 1907.

19. La «Chronique littéraire» de Maurice Gauchez, publiée à la parution du Roman de Pénéloppe (1952) dans un journal que nous n'avons pas pu identifier, a été découverte dans un cahier réunissant les articles qui concernent Emma Lambotte, consultable aux AMVC de la Letterenhuis d'Anvers, sous la cote L 1604.

20. Idem.

21. Les correspondances de Lambotte avec Mockel et Elskamp sont disponibles aux AML de la Bibliothèque royale, respectivement sous les cotes ML 3090 et ML 5454. 
Emma Lambotte tient des rubriques littéraires et artistiques dans la presse liégeoise (Le Tout Liège, La Meuse) et anversoise (Le Matin, Le Méphisto). Elle y expose également ses récits de voyage en Égypte, au Canada et aux États-Unis. Elle offre sa collaboration à des revues artistiques bruxelloises, anversoises, françaises comme La Nervie, La Gerbe, La Renaissance d'Occident et La Revue francobelge. En 1910, sous le conseil de Laurent Tailhade, elle abandonne le pseudonyme d'Emaël pour publier son premier recueil poétique chez Vanier, à Paris : Les Roseaux de Midas. Le succès est immédiat, en grande partie grâce à la préface de son éminent protecteur. Dans le Courrier d'Anvers du 18 novembre 1910, on peut lire :

Je vous avoue que lorsque je reçus le livre que Mme Emma Lambotte publie chez Vanier et que Buschmann a typographié avec le goût qui le caractérise, je fis la grimace. J'aime assez peu les femmes qui peignent et les femmes qui écrivent car j'ai toujours l'impression qu'elles n'ont pas de ménage, pas d'enfants ou pas d'amant. Mais l'étincelante préface de Laurent Tailhade qui sert de frontispice au volume me charma infiniment et dès les premiers feuillets des Roseaux, je fus conquis.

C'est dans cette ambiance anversoise et interculturelle où tout semble lui réussir qu'Emma Lambotte publie deux traductions françaises d'auteurs wallons chez Robert Protin, la maison d'édition de son frère. Écrits wallons rassemble des contes et chroniques de François Renkin (1872-1906), un auteur wallon décédé quelques années plus tôt. En présentant sa traduction, illustrée par le peintre liégeois Auguste Donnay, comme une tentative de faire connaître l'écrivain en dehors de la Wallonie, Lambotte participe au processus de propagation de la littérature wallonne en traduction. Elle accompagne d'ailleurs la publication d'une série de conférences aux quatre coins de la Belgique :

Madame Lambotte ne s'est pas bornée à ce pieux hommage à son compatriote : elle a résolu de faire connaittre Renkin en Belgique, même en Flandre, par des conférences. L'œuvre traduite de Renkin a donc été lue et commentée devant un public chaleureux à Charleroi, à Liège, à Verviers, à Ostende, peut-être ailleurs. (Feller, 1913, p. 128)

En donnant ainsi de sa personne, elle contribue également à la création nostalgique d'un imaginaire partagé : «j'ai retrouvé mon petit pays, j'ai mieux compris le sentiment de la patrie» (Lambotte, 1912, p. 5), une posture louée dans Le Tout Liège: «Mme Lambotte nous apportait une belle leçon de confiance en nous même; les Liégeois, 
nous dit-elle, doivent rechercher et aimer tout ce qui, chez eux, est digne d'être aimé» (Le Tout Liège, décembre 1912 22 . Elle compare également la Flandre et la Wallonie dans leur façon d'accueillir les poètes locaux :

Les poètes wallons, dit Mme Lambotte, sont peu connus. Nous ne faisons rien, en Wallonie, pour les produire; il y a chez nous de la négligence, un manque de confiance inexcusable. En Flandre, on fait une réclame tapageuse au moindre écrit du plus petit littérateur flamand. (Le Soir, décembre 191223)

Si on en croit la réception wallonne de ces traductions, l'identité wallonne d'Emma Lambotte, qui habite pourtant la Flandre depuis presque vingt ans, ne fait aucun doute; c'est « une liégeoise qui habite Anvers, une luronne très wallonne, qui ne se laissera pas aisément flamandiser»(Feller, 1913, p. 128); «Mme Emma Lambotte, qui vit loin de Liège, aime sa ville et son pays wallon avec une âme de poète et un bel enthousiasme d'artiste; sa conférence ne nous révélait pas seulement François Renkin, elle nous révélait aussi une Wallonne» (Le Tout Liège, décembre 1913).

La première partie du recueil reprend les originaux, la seconde, les traductions. Aucune explication ou note de bas de page ni aucun commentaire philologique n'accompagne les transpositions, qui ne sont là que pour servir l'original. Dans les journaux anversois et liégeois, la réception est bonne, mais, dans la plupart des cas, elle est imbibée de stéréotypes associés aux écrits dialectaux : naïveté, saveur, couleur et simplicité; "Mme Emma Lambotte a traduit l'œuvre de Renkin en une prose simple, qui garde toute la saveur saine et franche du wallon» (Le Tout Liège, décembre 1912). Ainsi, la traduction de Lambotte maintient les relations de pouvoir asymétrique entre les langues, le wallon ne pouvant être que simple, folklorique et sans prétention : «Au point de vue du folklore, du document, il a certes bien fait d'écrire en ce vieux langage roman si plein de couleur et de spontanéité; mais c'est cependant parce qu'il écrivit en wallon qu'il n'est pas plus connu» (Lambotte, 1912, p. 8).

En 1916, Emma publie une autre traduction - qu'elle assure avoir réalisée avant la guerre. Cette fois-ci, il s'agit de l'œuvre de

22. Fait partie des articles réunis dans un cahier consultable aux AMVC de la Letterenhuis d'Anvers, sous la cote L 1604.

23. Fait partie des articles réunis dans un cahier consultable aux AMVC de la Letterenhuis d'Anvers, sous la cote L 1604. 
Nicolas Defrecheux (1825-1874), un auteur wallon de renom déjà traduit dans plusieurs langues ${ }^{24}$. La fonction de la traduction est par conséquent différente, et cela se ressent dès la préface.

$\mathrm{Au}$ moment où tout ce qui faisait la caractéristique des vieilles provinces semble devoir se perdre et s'uniformiser; où les langues secondaires, les langues de terroir, disparaissent pour se fondre en une plus grande unité linguistique et administrative, ce qui est le cas pour la Provence et la Bretagne autant que pour notre Wallonie, des poètes surgissent qui continuent la tradition orale. Ils la continuent, malgré ce que l'on appelle, quelquefois fort mal à propos, «le progrès des lumières». (Lambotte, 1916, p. 7)

Étude sur Nicolas Defrecheux, et traduction de son œuvre, illustrée par Donnay et Rassenfosse, ne s'insère plus dans un élan de partage et de découverte, mais dans une revendication identitaire défensive : «Le génie en wallon, est encore du génie» (ibid., p. 18). En traduisant une sélection de poèmes, boutades, conseils, contes et mœurs wallons, Emma trace en outre un lien «naturel», «essentiel» entre les histoires et valeurs ancestrales wallonnes et les lecteurs d'aujourd'hui. Pourtant, cette fois, hormis quelques manuscrits de l'auteur insérés ici et là dans le recueil, il n'y a plus de place pour la langue wallonne. Emma résume, explique, commente et interprète :

Defrêcheux réveille en nous l'amour de notre sol, l'amour de notre langue; car nous avons beau être sceptiques, le patriotisme, l'héroïsme même, ne sont pas morts en nos cœurs. Nous sommes patients; mais, si nous avons bon cœur, nous avons aussi mauvaise tête! Nous sommes endurants, mais que l'on ne s'y fie pas trop! Liège est encore «la cité ardente»! s'il nous fallait entreprendre un nouveau 1830, ce ne serait plus un air de la Muette de Portici qui nous animerait, mais un vieil air wallon et des paroles de Defrêcheux. (ibid., p. 49)

Dissimulée derrière la langue traduisante, la langue source est présentée comme complexe et intraduisible au travers des nombreuses notes de bas de page et dans les commentaires qui peuplent la tra-

24. «On connait Defrêcheux à l'étranger; certaines de ses œuvres furent traduites en allemand et en flamand. Jules Feller, notre éminent philologue académicien, transcrivit même "Leyi 'm plorer" en vers latin. Mme Lambotte sentit qu'il manquait une importante traduction au tableau de Nicolas Defrêcheux. Elle écrit dans sa préface : "Trop peu de poèmes furent rendus en français. Il y avait-là, me semble-t-il, une lacune à combler. Croyant bien faire, j'ai traduit en cette langue toutes les œuvres du poète. Je les destine surtout aux Français et aux Flamands de bonne volonté.” Puissent ceux-ci l'avoir compris!» (Rousseau, 1937, p. 59). 
duction - «j'étais rouge comme une cressôde [note de bas de page : cressôde est intraductible. Cressôde ou crissôde est une variété cultivée, à fleurs doubles, de la marguerite des près]» (ibid., p. 100). De cette manière, elle dresse une barrière entre le français et le wallon. Comme l'annonce la préface, cette œuvre a pour objectif de défier l'unité linguistique - en surface uniquement, bien sûr, puisque le texte est français - et avec elle l'unité administrative belge. La traduction de Mes deus Lingadjes en est un excellent exemple.

Mes deux langues $\underline{25}$

J'emploie pour parler

Et même pour penser,

Deux langues apprises dans ma jeunesse.

L'une se nomme le français;

Toujours j'admirerai

Sa douceur et ses richesses.

L'autre c'est le wallon;

Rude et franc comme nos pères

C'est un vert rameau qui s'étend sur la libre terre.

Ah je vous aime langue de ma patrie

Vieux Wallon charmez mes oreilles

Jusqu'au dernier jours de ma vie

Pour apprendre le français,

Je me suis appliqué

Bien des heures sur les bancs de l'école;

J'étais tourmenté

Car, de mes libertés,

On ne m'en avait laissé aucune.

Le wallon! Je l'appris

En jouant à la toupie

Au cerf-volant, aux billes

En plein air, sous le ciel bleu

[...]

La langue française

Toujours, nous servira

On la parle dans le monde entier;

Grâce à elle, dirai-je

25. Defrecheux (1861), Mes deus Lingadjes. Djêplôye po djâzer/Et minme po tûzer/ Dêus lingadjes apris d' djonèsse/ Onk si nome francès : / Todi dj'admeûr'ré/ Si doûceûr èt sès ritchèsses / L'aute, c'ès-st-on walon/R Rude èt franc tot come nos péres/ C'è-st-on vért djéton/Qui s'sitind avâne lîbe tére!/ A! dji v's-Inme, lingadje di m'patrèyel Vî Walon, hossîz mès-orêyes/Djusqu'â dierain djoû di m'vèye (etc.). 
Tous les peuples se tendent

La main par-dessus les frontières.

Mais si, contre nos droits,

Se dressait un Téméraire

Notre Wallon dirait :

Aux armes! Liège et Saint Lambert ${ }^{26 !}$ (ibid., p. 48-49)

La réception de la traduction d'Emma Lambotte est ambivalente. Son ancienne professeure, la Baronne de Waha, la félicite pour service rendu à la patrie wallonne, mûre pour l'autonomie, tandis qu'un chroniqueur de la revue La Wallonie voit dans sa traduction une façon de rendre hommage à la Belgique unie. Pour la première, le lien entre littérature et autonomie politique est évident :

Je vous félicite d'employer votre talent d'écrivain au service de notre Patrie Wallonne. Votre traduction des poésies de Defrêcheux contribuera à révéler à l'étranger l'existence d'un peuple wallon, fier de ses souvenirs historiques et d'une langue pittoresque dont la littérature, depuis un siècle, prend un essor inattendu. Tout dénote que nous sommes mûrs pour l'autonomie administrative dont nous poursuivons la réalisation. (Lettre de Waha à Lambotte, le 14 janvier 1917, op. cit. Lambotte, 1927, p. 9)

Pour le second, la traduction d'Emma Lambotte permet au peuple belge de découvrir un nouveau poète qui conte, en wallon, les mêmes joies et peines que ses compatriotes :

Il est, plus spécialement, un champ d'action où le Belge intellectuel est tenu de s'employer en vue de réaliser la communion si nécessaire des âmes dans le culte de la patrie une et indivisible : révéler au peuple combien ses littérateurs et ses poètes, qu'ils soient d'expression française, flamande ou wallonne ont pu conter ou chanter avec noblesse et talent son histoire, ses communes vertus, ses joies et ses souffrances partagées, ses aspirations. Telle est la noble tâche que poursuit Mme Lambotte en vulgarisant l'œuvre de M. Defrêcheux. (La Wallonie, avril $1920^{27}$ )

C'est encore à Anvers qu'Emma Lambotte publie Petits poèmes traduits de l'Iroquois (1918). Dans ce recueil de vers libres teintés de féminisme, la poétesse s'imagine traductrice de l'intraduisible langue iroquoise. Par le biais d'«un sentiment qui s'appelle iroquois et qui n'avait pas été défini jusqu'à ce jour» (lettre d'Elskamp à Lambotte,

26. Évêque martyr, symbole de la ville de Liège.

27. Fait partie des articles réunis dans un cahier consultable aux AMVC de la Letterenhuis d'Anvers, sous la cote L 1604. 
10 août 1918), elle veut supprimer toute médiation entre la sensation et l'expression :

Il faut que l'on voie clair en soi;

En soi... et dans autrui.

Or, autrui n'a-t-il pas aussi un indéfinissable cœur?

Tout comme toi,

Benoît lecteur.

Tâche d'y lire!

Hélas comment faire pour traduire

De l'intraduisible iroquois? (Lambotte, 1918, p. 16)

Dans la Défense wallonne du $1^{\text {er }}$ juin 1924, Jeanne Gosselin écrira :

C'est à l'époque où elle habitait Anvers, (Anvers, dit-elle quelque part, où il fait si flamand!) et peut-être pour obéir à un obscur besoin de réaction contre la triste ambiance, que Mme Lambotte eut la jolie idée de traduire en français les œuvres de François Renkin d'abord, de Nicolas Defrêcheux ensuite. ${ }^{28}$

En tous cas, force est de constater que cette «triste ambiance» lui a été largement profitable et lui a fourni un terreau interculturel et multilingue propice à l'expérimentation via la traduction linguistique et culturelle.

\section{De retour à Liège (Esneux, 1918-1928)...}

Le climat d'après-guerre est patriotique, germanophobe et antiflamingant. De retour à Liège, Emma Lambotte et son mari entrent en possession d'un petit château dans le village d'Esneux, à quelques kilomètres de la cité ardente. Albin, qui s'est distingué pendant la guerre en s'occupant des invalides, continue à exercer sa chirurgie révolutionnaire à Anvers. Le couple voyage, notamment en GrandeBretagne, où naît leur fille unique, Marie Ange Good, en 1920.

Après avoir abandonné le projet de réaliser une anthologie d'écrits wallons ${ }^{29}$, Emma s'engage sans concession dans le féminisme et dans la lutte wallonne contre la Flandre qui l'avait accueillie quelques années plus tôt. Figure publique, elle prend la parole lors des réunions contre le flamingantisme, publie des essais sur la question linguistique, préside l'Union des femmes de Wallonie et prend part à l'Internationale des femmes. Dans les années 1920, la question

28. Fait partie des articles réunis dans un cahier consultable aux AMVC de la Letterenhuis d'Anvers, sous la cote L 1604.

29. Dans une lettre datée du 10 août 1918, Elksamp déplore le départ d'Emma et discute de son projet d'anthologie wallonne. 
de la flamandisation de l'Université de Gand divise la nation. Pour Emma Lambotte, le bilinguisme franco-wallon devient le symbole de l'opposition au bilinguisme franco-flamand d'État et un signe identificatoire par excellence : «Nous sommes bilingues : françaiswallon et, comme l'a dit un orateur notoire : "Nos n'sèri pu wallons si nos nos leyî fé" [nous ne serions plus wallons si nous nous laissions faire] (ma trad.)» (Lambotte, $\left.1921^{30}\right)$. «Pas de bilinguisme françaisflamand en Wallonie! Notre bilinguisme, à nous : français-wallon, nous suffit. Ce sont-là nos deux langues familiales, celles dont a si bien parlé notre grand poète populaire Nicolas Defrêcheux» (Lambotte, 1927, p. 89). Elle retourne maintes fois à Anvers pour y donner des conférences sur les auteurs wallons; ses traductions sont alors recyclées à des fins politico-linguistiques.

En mars 1920, année où le ministre des arts et des sciences Jules Destrée la nomme Chevalier de l'Ordre de Léopold (à titre littéraire), elle se rend au cercle artistique d'Anvers pour parler de Nicolas Defrêcheux. Le discours de l'enfant du pays, «notre concitoyenne, maintenant fixée à Esneux» (Le Matin d'Anvers, vers le 30 mars 1920), est accueilli chaleureusement. Dans une salle bondée, elle exprime sa joie de se retrouver dans sa «ville adoptive»( $L e$ Journal d'Anvers, vers le 30 mars 1920) où règne, selon Elskamp, un «flamingantisme insoutenable» (Elskamp à Lambotte, le 13 février 1922). L'évènement est clôturé par le chant des Liègeois, repris en cœur par l'assemblée, tandis qu'Emma reçoit des fleurs des organisateurs anversois et liégeois. Cependant, nous dit un chroniqueur de la revue La Wallonie, «ces fleurs de la Wallonie avaient encore pour moi une autre signification [...] C'est que je déplorais que l'occasion de les offrir avait dû dépendre de l'intercession d'un aimable voisin!». Il poursuit :

Ce n'est pas la peine, en vérité, de nous appeler la «Wallonie», d'être, dans la première cité du pays, les défenseurs les mieux désignés du génie wallon, si nous devons laisser à d'autres le soin de révéler ou de fêter nos grands hommes. Puisse le geste amical et tout de délicatesse d'ailleurs du Cercle Royal Artistique être un enseignement pour nos dirigeants. Il y a trente ans que nous sommes dans l'ornière; le motif est sans doute suffisant pour en sortir. (La Wallonie, avril 1920)

30. Emma Lambotte dans Le Journal des femmes de 1921. Fait partie des articles réunis dans un cahier consultable aux AMVC de la Letterenhuis d'Anvers, sous la cote L 1604. 
Durant toute la décennie, Emma mobilise ses forces pour «sortir de cette ornière» en exposant, à qui veut l'entendre, son point de vue sur la question linguistique en Wallonie, en Flandre, mais également en France, comme dans revue nantaise La Gerbe :

On croit généralement à l'étranger que les Belges parlent deux langues : le français, élu par la bourgeoisie, l'aristocratie et les gens tant soit peu instruits, et le flamand, ronron du peuple. Mais on ignore trop souvent qu'il y a encore le wallon. [...] Nous ne forçons pas les Flamands à l'étude du wallon, qu'ils ne nous tourmentent pas davantage par l'étude de leur dialecte et, s'ils invoquent la question d'utilité, nous leur dirons qu'il serait bien plus utile de hausser leur peuple jusqu'à la langue française [...] La France s'attarde-t-elle à l'étude «officielle» du breton ou du provençal ? On en remonte pas le courant : la logique et la philosophie tendent à tout simplifier, à tout unifier. Moins il y aura de langues, mieux cela vaudra. (La Gerbe, avril 1920, p. 200-201)

En évoquant une conversation avec son domestique anversois, elle retourne les prêts-à-penser sociaux et réinvente la diglossie belge, en mettant wallon et flamand sur le même pied et en approuvant le rapport de force imposé par la nation belge :

Nous avions un domestique anversois; il avait le bon esprit de parler français et me disait : «Les Wallons parlent wallon et français, nous parlons flamand et français et, grâce au français, tous les Belges se comprennent ». Mais ce garçon nétait ni un activiste ni un arriviste et il avait du jugement. Je lui répondais : «les flamingants veulent nous imposer leur langue; leur imposons-nous le wallon, nous autres? Ce serait un beau «tolle» si nous les forcions à l'apprendre!» (La Gerbe, avril 1920, p. 202)

\section{... puis à Anvers (Wilrijk 1928-1963)}

En 1928, Emma retrouve son mari à Anvers. Le couple s'installe à Wilrijk, qui est alors un petit village près de la métropole. Son ami Jules Feller s'inquiète de ce nouveau déménagement et de l'influence de celui-ci sur l'engagement de son amie.

Bien que le monde soit petit, aux dires des philosophes, des culs-deplomb qui ne le mesurent pas avec leurs jambes, je trouve Anvers si éloigné qu'il me semble toujours que vous vous êtes retirés du monde. Esneux me paraissait beaucoup plus accessible. [...], je me demandais si vous aviez renoncé au français, au wallon, en faveur de l'Antwerpsche taal. Mais votre lettre me rassure. Vous continuez à 
écrire, puisque vous me demandez de relire votre manuscrit. (Feller à Lambotte, le 21 décembre $1929^{31}$ )

Les craintes de Feller sont fondées. De retour en Flandre, l'ardeur wallonne d'Emma Lambotte faiblit. Elle abandonne peu à peu sa lutte contre le flamingantisme pour se concentrer sur l'art, la littérature et le féminisme. Est-ce parce que, de retour à Anvers, elle n'ose plus militer contre son réseau? Est-ce parce qu'elle craint les réactions de son entourage anversois? Est-elle découragée par l'échec des revendications wallonnes? La phase finale de la néerlandisation de l'Université de Gand en 1930, puis l'implémentation de lois linguistiques en faveur du flamand dans les écoles primaires et secondaires avaient en effet marqué un tournant dans la question linguistique ${ }^{32}$. Elle publie encore néanmoins quelques écrits nostalgiques sur la Wallonie. Elle se distingue alors de ses collègues qui ont pris l'habitude de chanter le plat pays, les kermesses et les paysages de Flandre en adoptant la posture de l'exil, par exemple dans Heureux Vallon (1934).

En exil, ô mon Dieu, ne me laisse mourir!

Car mon natal pays, tout en fines nuances

Est pareil à la douce France ${ }^{33}$

Les bonnes gens y sont autant d'autres moi-même :

Ils m'entendent, ils m'aiment,

Et moi, je les comprends [...]

J'ai tant besoin de l'atmosphère wallonne

Des visages rieurs

Du doux,

Du chantant parler de chez nous!

J'en ai besoin pour être aisément bonne;

D'autres accents trop durs ont blessé mon

oreille... (Lambotte, citée dans Rousseau, 1937, p. 40)

31. La correspondance entre Lambotte et Feller est disponible aux AML de la Bibliothèque royale, sous la cote ML 5155.

32. Mentionnons notamment la loi de 1921 qui impose l'utilisation exclusive du néerlandais dans l'administration en Flandre et la loi de 1932 régulant l'utilisation du néerlandais dans l'enseignement primaire et moyen de Flandre.

33. L'association de la Wallonie à la France, plutôt qu'à l'État belge unitaire, est de plus en plus fréquente dans l'entre-deux-guerres. En 1937, le biographe de Lambotte écrit : «En elle, le sang wallon se mêle au sang français. Les caractéristiques psychologiques des deux races se retrouvent dans ses écrits. Du wallon, elle possède l'esprit de pénétration, le caractère frondeur, le verbe gai [...]. Du français, elle montre la sensibilité, la grâce mutine, le sens des images, la fraîcheur d'expression» (Rousseau, 1937, p. 1). 
Elle assimile ensuite progressivement le profil de l'élite belge francophone, celui qu'elle avait déjà laissé entrevoir avant de déménager, dans son essai biographique sur la militante wallonne Léonie de Waha, paru en 1927. Si cette étude avait encore une portée politique et participait à un processus de canonisation des "grandes Wallonnes", elle ne partageait pas l'avis de son mentor dans La Barricade du 30 décembre 1922, qui proposait d'accepter la néerlandisation de l'Université de Gand, de transposer les cours français à Tournai et de ne laisser aux Flamands «que le flamand». Emma Lambotte jugeait cette option injuste, car elle ne pourrait satisfaire l'élément «fransquillon ${ }^{34}$ » de Flandre, auquel elle appartenait désormais en tant que Wallonne fixée à Anvers. En 1935, elle publie un très patriotique Astrid, Reine des Belges et entre tant et si bien dans le moule de l'élite francophone anversoise que Roger Avermaete lui adresse un manifeste de défense du bilinguisme en Belgique. Emma le rejette poliment en rappelant à son collègue que «[1]es Wallons fixés en Flandre tâchent de comprendre et de répondre en langue thioise - par politesse - tout simplement» (Lettre de Lambotte à Avermaete, 16 septembre 1938). En 1949, elle publie Les Prestiges d'Anvers (1949), qui synthétise l'atmosphère anversoise, décrit ses monuments, ses églises et ses peintres. Ce dernier ouvrage est merveilleusement accueilli par la presse néerlandophone, qui assimile définitivement son auteure à la ville :

Van de Luiker, doch sedert vele jaren te Antwerpen gevestigde Franstalige schrijfster, Emma Lambotte, verscheen zo pas een klein, doch verrukkelijk boekje. "Prestige d'Anvers» waaruit op iedere bladzijde de grote liefde spreekt, die zij de Scheldestad toedraagt (...) een soort van geloofsbelijdenis van een lyrisch georiënteerde vrouw, die volledig het Antwerps klimaat heeft geassimileerd. (Volksgazet du 3 novembre 1949)

[Liégeoise, mais écrivaine francophone fixée à Anvers depuis longtemps, Emma Lambotte a publié un petit livre exquis. "Prestige d'Anvers» évoque, sur chaque page, le grand amour, celui qu'elle porte à la cité de l'Escaut [...] une sorte de profession de foi de la part d'une femme lyrique, qui a totalement assimilé le climat anversois.] (ma trad.)

Entre-temps, elle continue à jouer la «mécène accomplie [qui] collectionne les œuvres des maîtres peintres les plus discutés. Sa galerie,

34. Fransquillon (contraction de Frans [le français] et nobiljon [noble]) désigne une classe socioéconomique dominante en Flandre, mais également en Wallonie (Françkillon) dont le français est le signe identificatoire. 
fleurant musée, fait florès à Anvers» (Ensor, 1936, cité dans DerreyCapon, p. 321); elle est régulièrement invitée à s'exprimer aux BeauxArts d'Anvers pour parler peinture (p. ex. en janvier 1947, sur James Ensor) et voyage. Celle que James Ensor aura appelée «Reine Flandre, Reine wallonne» meurt à Wilrijk en 1963, huit ans après son mari.

\section{Conclusion}

Le cas d'Emma Lambotte est bien entendu trop limité pour tirer des conclusions générales. Il faudrait appliquer le même genre d'analyse à d'autres acteurs et institutions afin de dresser un panorama représentatif des interactions complexes qui ont eu lieu en Belgique durant cette phase de transition linguistique et culturelle. Revenons néanmoins aux considérations de départ. Emma Lambotte décline un profil qui, bien que cohérent en soi, n'entre dans aucune des catégories réductionnistes de l'historiographie belge. Dans ses allersretours entre Anvers et Liège, elle a d'abord tenté de propager et d'ouvrir la culture et la langue wallonnes à l'épreuve de l'autre, qu'il soit flamand, francophone ou français, avant de se lancer dans une quête politico-linguistique vaine, puis de se résigner, laissant béante une faille que la Wallonie peine encore à combler. Elle nous a néanmoins permis d'entrer dans la boîte noire et de dégager quelques pistes de réflexion locales et globales.

1/ Les langues et cultures en Belgique ont été, dans la première moitié du $\mathrm{XX}^{\mathrm{e}}$ siècle, sujettes aux déplacements et aux transformations. L'approche binaire, franco-flamande, a largement dissimulé la composante wallonne et ainsi favorisé la propagation de toute une série d'amalgames $($ Wallonie $=$ Wallon = francophone; Flandre $=$ Flamand $=$ néerlandophone $)$ qui continuent à obstruer la compréhension des enjeux identitaires et sociolinguistiques de l'époque. Dans ses allers-retours entre Anvers et Liège, Emma Lambotte a combiné allégrement tous ces profils ainsi qu'une série de rôles de médiation (mécène, traductrice, conférencière, chroniqueuse, etc.), qui offrent un panorama riche et compréhensif de la configuration linguistique et culturelle belge.

2/ Les mouvements flamands et wallons, et avec eux les nombreux mouvements régionalistes de la fin du XIX ${ }^{\mathrm{e}}$ siècle, méritent d'être étudiés de façon comparative, mais également dans leurs intersections. Tout d'abord parce que, au service d'une lutte sociale, culturelle puis politique, les mouvements flamands et wallons ont tous deux tenté d'émerger au sein d'un État belge unitaire qui subordonnait 
leurs aspirations (linguistiques et culturelles, notamment) à celles de la nation. Ensuite, parce qu'ils nous offrent des interventions locales similaires qui ne résultent pas dans les mêmes destins. Ceci ne revient évidemment pas à dire que les inputs sont identiques. Une multitude de facteurs historiques, culturels, économiques et linguistiques qui dépassent largement les frontières (intra)nationales de la Belgique doivent être pris en compte. Par exemple, la parenté linguistique (romane) entre les dialectes de Wallonie et le français peut expliquer la relation moins conflictuelle des Wallons vis-à-vis de la langue dominante. Il n'empêche que la comparaison de ces mouvements permet de mieux appréhender l'émergence identitaire et son rapport avec les échanges interculturels. La Wallonie aurait-elle, moins bien que la Flandre, ménagé son auto- et hetero-représentation? C'est en tout cas ce qu'Emma Lambotte semble insinuer. Il faut également noter que l'autonomie administrative rêvée par Emma Lambotte, tout comme par Léonie de Waha, à un moment où la Wallonie aurait pu, économiquement, assurer seule sa survie, ne se réalisera que quand celle-ci sera en déclin économique et culturel (1980) et que la Flandre prendra les rênes de l'économie belge.

3/ La seule opposition entre monolinguisme et bilinguisme doit être remise en question. Il est pertinent de constater que, aux yeux des Wallons, le bilinguisme d'État apparaît comme une menace au bilinguisme régional et au plurilinguisme national. C'est ainsi qu'Emma Lambotte remet le bilinguisme en perspective en dressant un lien avec d'autres minorités linguistiques européennes. Dans le cadre belge, Lambotte n'envisage à aucun moment de mettre le wallon et le français sur le même pied, mais imagine plutôt une double configuration diglossique, où français d'un côté et flamand et wallon de l'autre, remplissent des fonctions sociétales différentes ${ }^{35}$. Anversoise, elle ne veut pas d'une Flandre monolingue et défend avec ardeur la position fransquillonne, celle de l'élite socioculturelle flamande, mais également de toute une série d' «immigrés» wallons en Flandre. Liégeoise, elle refuse l'extension d'une langue qu'elle voit, à l'instar de sa propre langue maternelle, comme un dialecte de faible rayonnement. Néanmoins, au fil des décennies, le wallon apparaît

35. À quelques exceptions près, le mouvement wallon s'est toujours défendu de vouloir substituer à l'usage du français celui d'un wallon unifié devenu langue officielle en Wallonie. À ce sujet, L'Áme wallonne croit utile de préciser que «la Ligue wallonne ne veut ni du flamand, ni du wallon, comme langue officielle véhiculaire ( (Pirotte, 1997, p. 184). 
de plus en plus, face au flamand, comme «hyper-minoritaire». Si «all languages are potentially minority languages» (Cronin, 1995, p. 87), toutes les langues sont également potentiellement des langues majoritaires.

4/ Le cas de la Wallonie nous offre un accès privilégié à l'histoire d'un échec, et par là même une vision plus complète des modalités de réussite. Quel rapport peut-on observer entre les développements culturels et identitaires et les transferts culturels, dont la traduction? La traduction ne fait-elle que reproduire les asymétries, participet-elle à la résistance, crée-t-elle des frontières ou fait-elle émerger de nouvelles configurations? Selon Gideon Toury, «translating may certainly serve as a means for both actual preservation and development of minority languages in their endeavor to 'resist displacement' by the corresponding majority languages » $(1985$, p. 7). Cependant, comme le rappelle Anthony Pym, «translation constructs cultural borders, no matter how many translators might be operating in the overlaps around those borders» (2010, p. 153). Les activités d'Emma Lambotte entre Anvers et Liège exposent également la créativité liée au déplacement, l'émergence de nouveaux mondes, de nouvelles combinaisons, de nouveaux réseaux. Par conséquent, constructions homogènes et procédés hétérogènes ou réductionnisme et complexité (Morin, 1990) s'entretiennent l'un l'autre dans une dynamique qu'on a encore du mal à saisir. Acceptons pour l'instant de voyager dans l'incertitude et de remettre en question les cloisonnements et catégories, qui ne montrent finalement qu'une infime partie du tableau.

\section{Références}

Anonyme (1898). «El'Walloneie». L'Âme Wallonne, 8 mars, n. p.

Balace, Francis (2012). «La Wallonie, ses cultures et l'Allemagne». In B. Demoulin, dir. Histoire culturelle de la Wallonie. Bruxelles, Fonds Mercator, p. 346-354.

Beck, Philippe et Hubert Roland (2013). «Lespace frontalier Meuse-Rhin entre nationalisme et internationalisme chez Josef Ponten et Friedrich Markus Huebner (1920-1940)». Revue belge de philologie et d'histoire, 91, p. 1239-1258.

Belgium.be. Informations et services officiels (s.d.). La Belgique, un État fédéral. Disponible à <https://www.belgium.be/fr/la_belgique/ pouvoirs_publics/la_belgique_federale> [consulté le 17/04/2019].

Buelens, Geert (2011). «Gotspe of godsgeschenk? Enkele notitites over zin en onzin van een Belgische literatuurgeschiedenis». In S. Vanasten et M. Sergier, dir. Literaire Belgitude. Bruggen en beelden/Vues du Nord. 
Louvain, Presses universitaires de Louvain, p. 67-80.

Cairney, Paul (2012). «Complexity Theory in Political Science and Public Policy». Political Studies Review, 10, p. 346-358

Chamberlin, William (2009). «Networks, Emergence, Iteration and Evolution ». Emergence: Complexity and Organization, 11, 4, p. 91-98.

Congrès Wallon (1906). Congrès Wallon : compte-rendu officiel. Liège, Thone.

Courtois, Luc et Jean Pirotte, dir. (1994). L'imaginaire wallon : jalons pour une identité qui se construit. Louvain-la-Neuve, Fondation wallonne P.-M. et J.-F. Humblet.

Cronin, Michael (1995). "Altered States: Translation and Minority Languages». TTR, 8, 1, p. 85-103.

Cronin, Michael et Sherry Simon, dir. (2014). The City as Translation Zone. Numéro spécial de Translation Studies, 7, 2.

D'hulst, Lieven, Marie Bourguignon, Koen Lemmens, Bieke Nouws, Heleen Van Gerwen et Reine Meylaerts (2016). "Les politiques en matière de traduction en Belgique de 1830 à 1914». In G. LaneMercier, D. Merkle et J. Koustas, dir. Plurilinguisme et pluriculturalisme. Des modèles officiels dans le monde. Montréal, Les Presses de l'Université de Montréal, p. 13-27.

Debouck, D.-J. (1909). «À propos de l'enquête sur la littérature nationale, par Sylvain Bonmariage». Le Thyrse, 11, p. 212-214.

De Geest, Dirk et Reine Meylaerts, dir. (2004). Littératures en Belgique : diversités culturelles et dynamiques littéraires/Literatuur in België. Culturele diversiteit en literaire dynamiek. Bruxelles, Peter Lang.

Delforge, Paul (2011). «Léonie De Waha ». Portail "Connaître la Wallonie», Wallons marquants. Disponible à <http://connaitrelawallonie.wallonie. $\mathrm{be} / \mathrm{fr} /$ wallons-marquants/dictionnaire/de-waha-leonie\#.Wq5OJDWzIV> [consulté le 16 mars 2018].

Derrey-Capon, Danielle, dir. (1999). James Ensor. Lettres à Emma Lambotte 1904-1914. Bruxelles, La Renaissance du Livre.

Espagne, Michel (2013). «La notion de transfert culturel». Revue Sciences/ Lettres, 1. Disponible à <http://rsl.revues.org/219> [consulté le 16 mars 2018].

Even-Zohar, Itamar (1997). "The Making of Culture Repertoire and the Role of Transfer». Target, 9, 2, p. 355-363.

Feller, Jules (1913). «Emma Lambotte». Wallonia, février, p. 128.

Francard, Michel (1996). «La légitimité linguistique passe-t-elle par la reconnaissance du statut de variété "nationale"? Le cas de la Communauté française Wallonie-Bruxelles ». Revue québécoise de linguistique, 26, 2, p. 13-23.

Francard, Michel (2013). Wallon, Picard, Gaumais, Champenois. Les langues régionales de Wallonie. Bruxelles, De Boeck.

Gross, Joan (2001). Speaking in Other Voices. An Ethnography of Walloon Puppet Theaters. Amsterdam/Philadelphie, John Benjamins. 
Hasquin, Hervé (1982). Historiographie et politique. Essai sur l'bistoire de Belgique et la Wallonie. Charleroi, Institut Jules Destrée.

Haust, Jean (1924). Pages d'anthologie wallonne. Paris, Éditions gauloises.

Kesteloot, Chantal (1993). "Mouvement wallon et identité nationale». Courrier hebdomadaire du CRISP, 1392, 7, p. 1-48.

Klinkenberg,Jean-Marie (1988). «Lidentité wallonne : hypothèques et faux papiers». La Revue Toudi, 2. Disponible à <http://www.larevuetoudi. org/fr/story/lidentit\%C3\%A9-wallonne-hypoth\%C3\%A8ques-etfaux-papiers> [consulté le 16 mars 2018].

Lambotte, Emma (s.d.). Correspondances conservées aux Archives et Musée de la Littérature (AML), Bruxelles.

Lambotte, Emma (s. d.). Archives et correspondances conservées au Archief en Museeum voor het Vlaamse Cultuurleven (AMVC), Anvers.

Lambotte, Emma (1912). Écrits wallons de François Renkin. Liège, Protin.

Lambotte, Emma (1916). Études sur Nicolas Defrêcheux, Poète wallon. Liège, Protin.

Lambotte, Emma (1918). Petits poèmes traduits de l'iroquois. Liège, Protin. Lambotte, Emma (1927). Léonie de Waha de Chestret. Liège, Imprimerie de La Meuse.

Lambotte, Emma (1934). Heureux Vallon. Paris, Les écrivains réunis.

Lambotte, Emma (1935). Astrid, Reine des Belges. Bruxelles, La Renaissance du Livre.

Lecours, André (2001). «Political Institutions, Elites, and Territorial Identity Formation in Belgium». National Identities, 3, 1, p. 51-68.

Leerssen, Joep (2014). «Networks and Patchworks: Communication, Identities, Mediators». In T. Lobbes et M. Gonne, dir. Belgian Cultural Mediators, 1830-1945. Crossing Borders, Borders Resisting. Revue belge de Philologie et d'Histoire, 92, 4, p. 1395-1402.

Lobbes, Tessa et Maud Gonne, dir. (2014). Belgian Cultural Mediators, 1830-1945. Crossing Borders, Borders Resisting. Revue belge de Philologie et d'Histoire, 92, 4.

Luyckx, Theo et Marc Platel (1985). Politieke Geschiedenis van België: van 1789 tot 1944. Anvers, Kluwer.

Marais, Kobus (2014). Translation Theory and Development Studies. A Complexity Theory Approach. Londres/New York, Routledge.

Marais, Kobus (2018). “Effects Causing Effects': Considering Constraints in Translation". In K. Marais et R. Meylaerts. Complexity Thinking in Translation Studies: Methodological Considerations. New York, Routledge. p. 53-72.

Meylaerts, Reine (2004). L'aventure flamande de la Revue belge. Bruxelles, P.I.E. Peter Lang.

Meylaerts, Reine (2009). «"Et pour les Flamands, la même chose” : quelle politique de traduction pour quelles minorités linguistiques?». Meta, 54,1, p. $7-21$. 
Meylaerts, Reine (2017). "Studying Language and Translation Policies in Belgium: What can We Learn from a Complexity Theory Approach?" Parallèles, 29, 1, pp. 45-59.

Meylaerts, Reine et Maud Gonne (2014). «Transferring the City Transgressing Borders: Cultural Mediators in Antwerp (1850-1930)». Translation Studies, 7, 2, p. 133-151.

Morin, Edgar (1990/2005). Introduction à la pensée complexe. Paris, Éditions du Seuil.

Moyaux, Adrien (2018). «Schéma d'une boîte noire: entrées, sorties et boîte au contenu inconnu». Disponible à <https://fr.wikipedia.org/ wiki/Fichier:Sch\%C3\%A9ma_d\%27une_bo\%C3\%AEte_noire.jpg> [consulté le 16 août 2019].

Pirotte, Arnaud (1997). L'apport des courants régionalistes et dialectaux au mouvement wallon naissant. Une enquête dans les publications d'action wallonne de 1890 à 1914. Louvain, Presses universitaires de Louvain.

Raxhon, Philippe (2004). «Le siècle des forges ou la Wallonie dans le creuset belge (1794-1914)». In B. Demoulin et J.-L. Kupper, dir. Histoire de la Wallonie. Toulouse, Privat, p. 246.

Roland, Hubert (2004). «Âme belge, entre-deux et microcosme : d'une fin de siècle à l'autre». Textyles, 24, p. 7-15.

Rousseau, Maurice (1937). Emma Lambotte. Bruxelles, Le livre belge d'aujourd'hui.

Sottiaux, Jules (1906). L'originalité wallonne. Paris, Édition artistique.

Toury, Gideon (2002). "Translation as a Means of Planning and the Planning of Translation: A Theoretical Framework and an Exemplary Case». In S. Paker, dir. Translations: (Re) shaping of Literature and Culture. Istanbul, Bogaziçi University Press, p. 148-165.

Van Nuffel, Robert (1994). "Emma Lambotte». In Nouvelle Biographie Nationale, 3. Bruxelles, Académie royale de Belgique, p. 212-215.

Werner, Michael et Benedict Zimmermann (2003). "Penser l'histoire croisée : entre empirie et réflexivité». Annales. Histoire, Sciences sociales, 1,58, p. $7-36$.

Wiener, Norbert (1952). Cybernétique et société. Paris, Deux-Rives.

Maud Gonne
F.R.S.-FNRS

Université de Namur et Université catholique de Louvain

Namur, Belgique et Louvain, Belgique maud.gonne@unamur.be 